\title{
Editorial
}

\section{Regional Coupled Model and Data Assimilation}

\author{
S. Zhang $\mathbb{D},{ }^{1,2}$ Y. Xie, ${ }^{3}$ F. Counillon $\left(\mathbb{D},{ }^{4}\right.$ X. Ma, ${ }^{1,2}$ P. Yu, ${ }^{5}$ and Z. Jing ${ }^{6}$ \\ ${ }^{1}$ Key Laboratory of Physical Oceanography, Ministry of Education, Ocean University of China, Qingdao, China \\ ${ }^{2}$ Qingdao National Laboratory of Marine Science and Technology (QNLM), Qingdao, China \\ ${ }^{3}$ Earth System Research Laboratory, National Ocean and Atmosphere Administration, Department of Commerce, Boulder, CO, USA \\ ${ }^{4}$ Nansen Environmental and Remote Sensing Center and Bjerknes Centre for Climate Research, Bergen, Norway \\ ${ }^{5}$ CICS/ESSIC, University of Maryland, College Park, MD, USA \\ ${ }^{6}$ Department of Oceanography, Texas A\&M University, College Station, TX, USA
}

Correspondence should be addressed to S. Zhang; szhang@ouc.edu.cn

Received 29 March 2018; Accepted 29 March 2018; Published 8 May 2018

Copyright (C) 2018 S. Zhang et al. This is an open access article distributed under the Creative Commons Attribution License, which permits unrestricted use, distribution, and reproduction in any medium, provided the original work is properly cited.

To further understand the mechanisms of local weather and climate anomalies and predict them skillfully, highresolution coupled modeling and data assimilation is necessary. On the one hand, regional coupled model and data assimilation is an important means to understand the details of local mesoscale and submesoscale air-sea interactions and how atmospheric and oceanic processes interact across multiple scales. On the other hand, societal needs require weather-climate studies to better resolve, evaluate, and predict regional changes/variations as well as extreme events. We need to detect how large-scale fluctuations influence the local weather and climate anomalies and how local weatherclimate perturbations feedback to large-scale background. To address these questions, weather and climate modeling must simultaneously resolve higher resolutions and local mesoscale and small-scale physical processes in increasingly greater details. Given the limitation of computing power, the dynamically downscaling framework that nests highresolution regional coupled models into a coarse-resolution global earth system model is an efficient way in such studies. While such a framework efficiently advances our understanding of the attribution and impact of large-scale phenomena on local conditions, it also provides an opportunity to link scientific advances with severe weather alerts at the local level. We expect that regional coupled models with well-designed boundary processing and coupled data assimilation can progressively advance climate sciences and promote local societal services.
In this special issue, we collect 8 papers that cover the regional observing system sampling and impact, quality control of observations in regional details, assimilation of nonconventional observations, improved assimilation method, and advanced assimilation strategy as well as parameter estimation for local regional phenomena.

How to configure the sampling points is an interesting topic in the regional observing system design. The paper titled "Optimal Configuration Method of Sampling Points Based on Variability of Sea Surface Temperature" uses the $K$-means clustering algorithm to optimize the sampling strategy based on historical SST observations. The gained results provide a new method for configuring sampling points of ocean in situ observation with limited resources. In the paper titled "Evaluation of the Impact of Argo Data on Ocean Reanalysis in the Pacific Region," the authors use the framework of Observing System Simulation Experiments (OSSEs) to evaluate the impact of Argo data on ocean reanalysis in the Pacific region. They found that the Argo temperature and T-S relationship play an important role in the analysis of this region while the direct adjustment from the Argo salinity observations is relatively trivial in the northwest Pacific if the Argo temperature data are used during the reanalysis. The quality control of observations is an important aspect of regional modeling and data assimilation. In the paper titled "A Quality Control Method Based on an Improved Random Forest Algorithm for Surface Air Temperature Observations," the authors combine the artificial fish swarm algorithm and the 
random forest regression function to apply quality control to surface temperature at multiple stations. Their work presents a promising method on surface air temperature observations for regional modeling and assimilation studies.

The assimilation of nonconventional observations is a very important aspect in regional weather and climate studies. Aircraft-based data are a promising source of abovesurface observations to be assimilated into mesoscale models. The paper titled "Assimilation of Aircraft Observations in High-Resolution Mesoscale Modeling" discusses the results of assimilating TAMDAR (the Tropospheric Airborne Meteorological Data Reporting) observations. The impact of TAMDAR observations in $1 \mathrm{~km}$ WRF (Weather Research and Forecasting) model is evaluated. They found that the regional forecast can be improved significantly with such aircraft observations although they only use a simple nudging scheme.

The assimilation method is a key point for the regional coupled model and data assimilation. In the paper titled "An Efficient T-S Assimilation Strategy Based on the Developed Argo-Extending Algorithm," authors present a new strategy to use the sea surface temperature (SST) to reconstruct T$S$ profiles and improve assimilation quality when in situ T-S profiles are insufficient. The paper titled "A Potential Density Gradient Dependent Analysis Scheme for Ocean Multiscale Data Assimilation" addresses the issue of how to maintain oceanic mixing along potential density surface in ocean data assimilation (ODA) and proposes a new ODA strategy. Their results show that the new scheme significantly improves the model forecast skills with more dynamically consistent information in initial conditions. In the paper titled "Formulations for Estimating Spatial Variations of Analysis Error Variance to Improve Multiscale and Multistep Variational Data Assimilation," the authors discuss the issue of constructing multiscale error covariance in variational data assimilation. Their method successively improves the variational assimilation quality, being very promising, although it is only demonstrated in idealized experiments.

The special issue also includes one paper discussing the problem of air pollution transport. In the paper titled "The High Order Conservative Method for the Parameters Estimation in a $\mathrm{PM}_{2.5}$ Transport Adjoint Model," authors apply Piecewise Parabolic Method (PPM), a high order and conservative interpolation, to the parameter estimation in a $\mathrm{PM}_{2.5}$ transport adjoint model. In this piece of interesting work, numerical experiments are taken to show the accuracy of PPM in space and its ability to increase the well-posedness of the inverse problem. In real case simulation experiments, the results are in good agreement with the observations from the 21th APEC Summit.

$$
\begin{array}{r}
\text { S. Zhang } \\
\text { Y. Xie } \\
\text { F. Counillon } \\
\text { X. Ma } \\
P . Y u \\
\text { Z. Jing }
\end{array}
$$



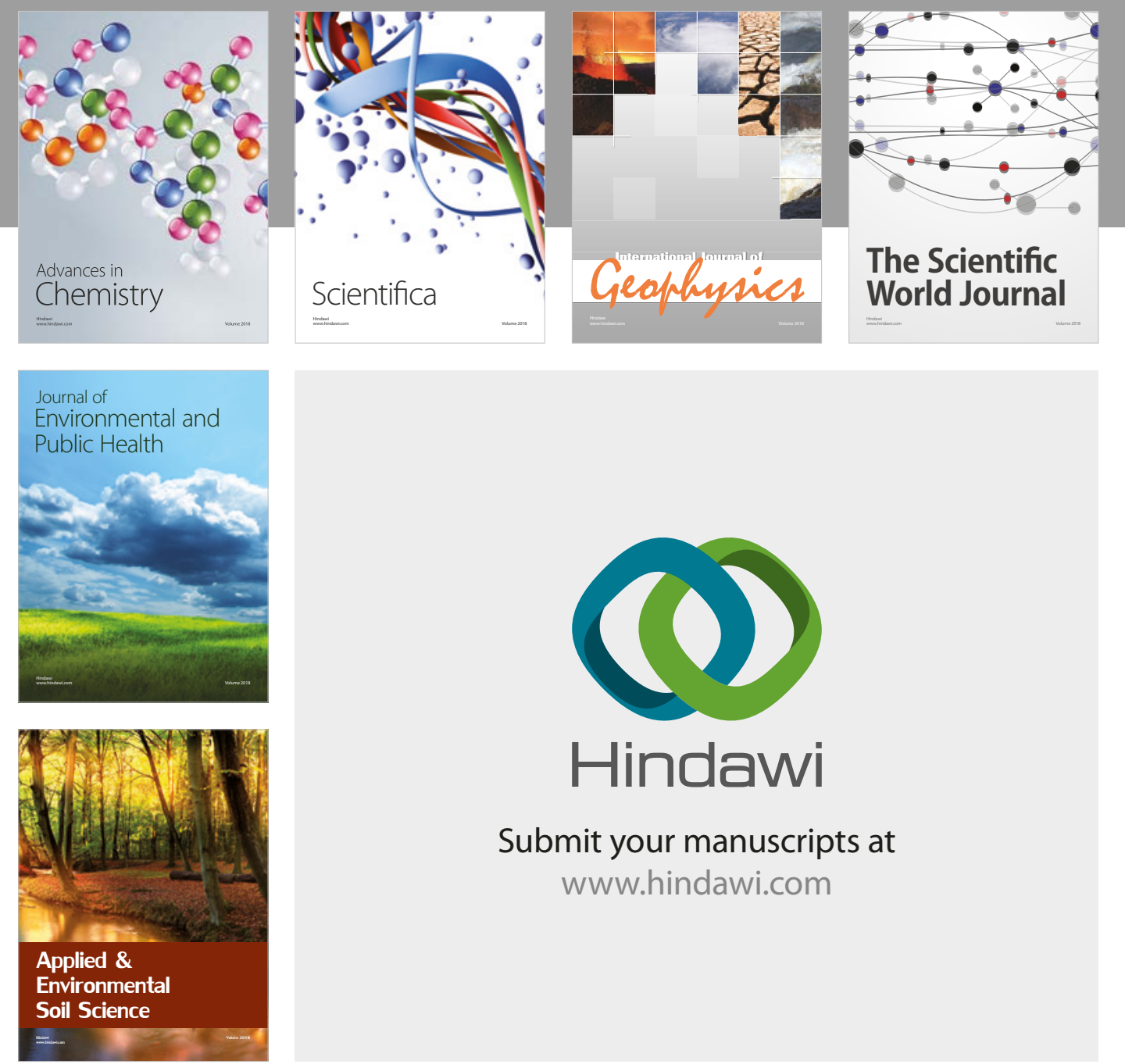

The Scientific

\section{World Journal}
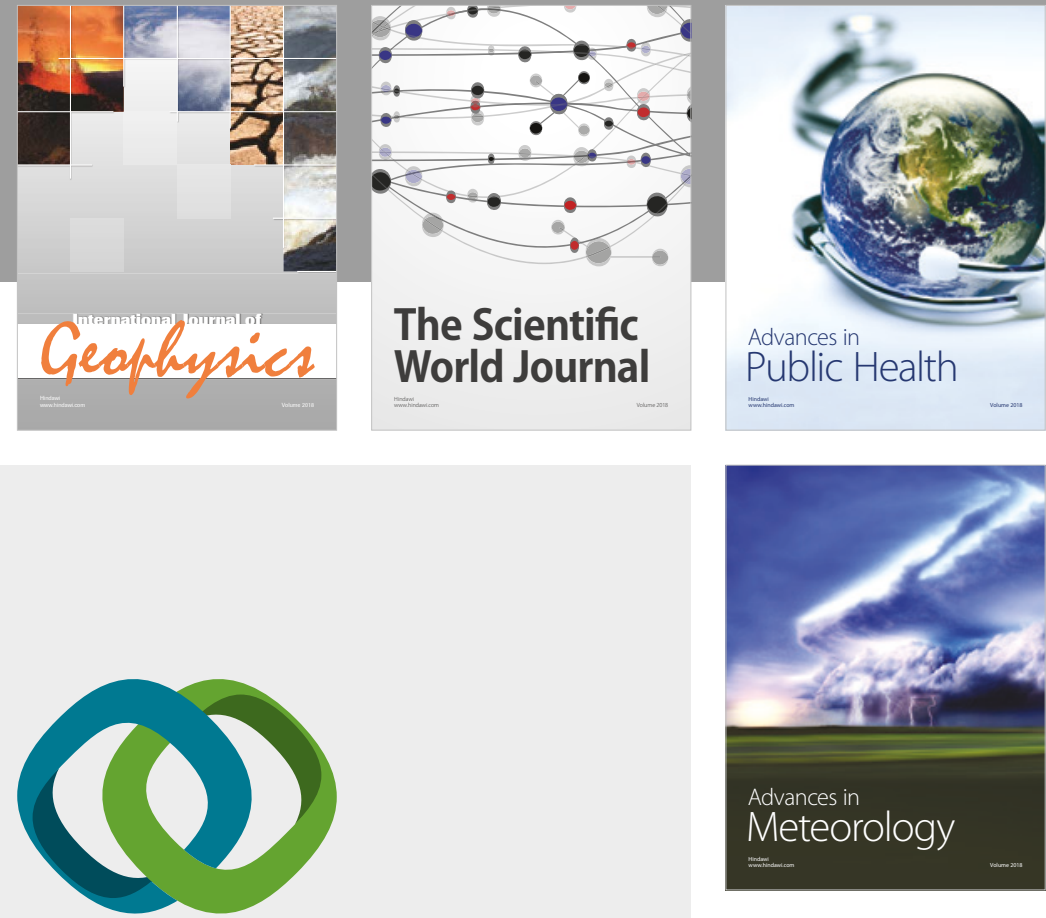

Advan

Public Health

\section{Hindawi}

Submit your manuscripts at

www.hindawi.com
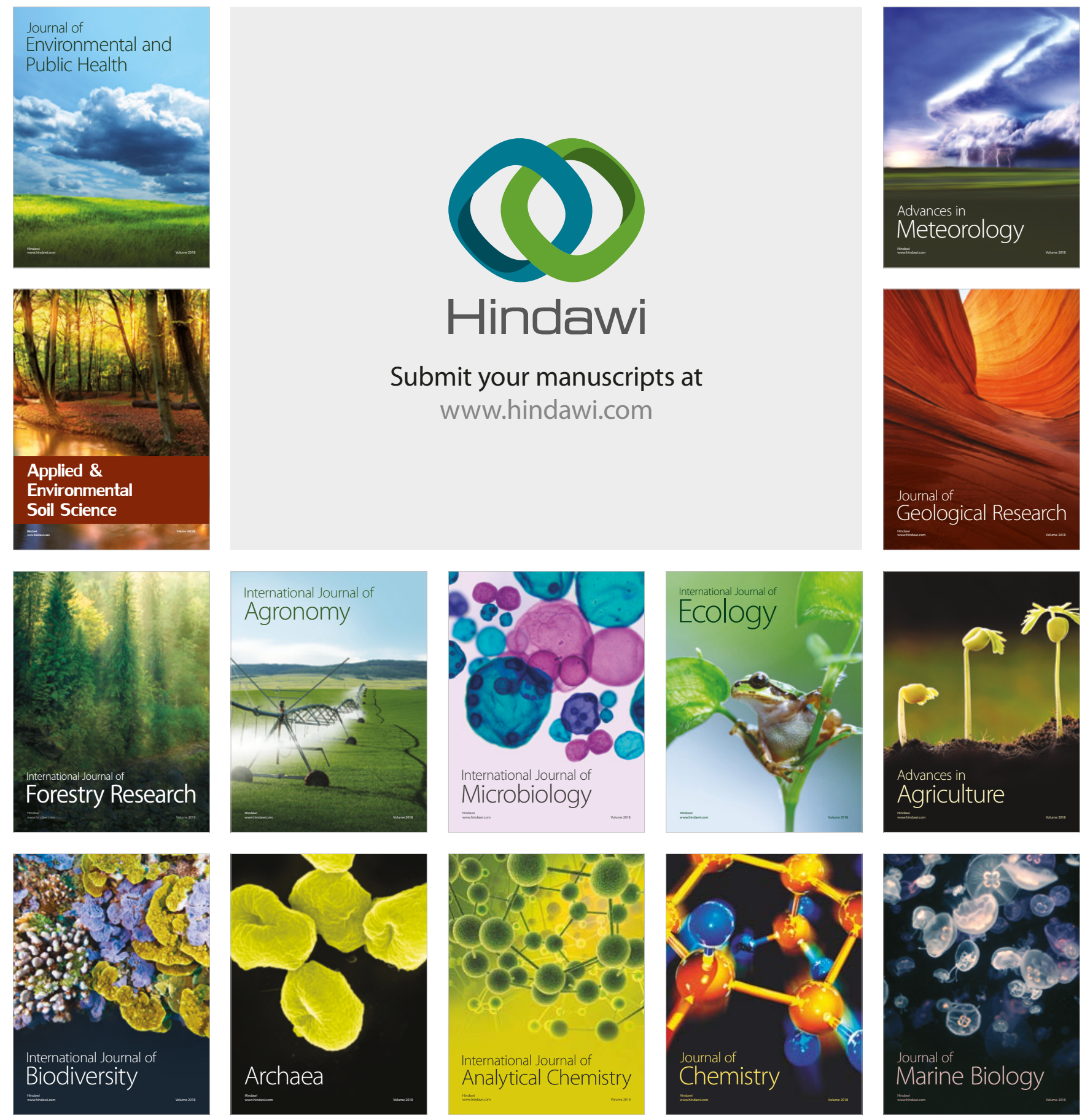